linear space determined by the columns of $\kappa^{*}$, and the conjugate of each row of $\lambda$ belong to the linear space of the columns of $\kappa$. This general reciprocal reduces to the ordinary reciprocal if $\kappa$ is non-singular. The general reciprocal of a (positive) Hermitian matrix is again (positive) Hermitian. It is not always true, however, that the reciprocal of a product is the product of the reciprocals in reverse order.

The book closes with a discussion of the Gramian matrix arising from a positive Hermitian bilinear equation, and the problem of orthogonalization with respect to such an operator. Every finite or infinite sequence of vectors can be orthogonalized with respect to the positive Hermitian bilinear operator $B$, and if $B$ is proper, the orthogonalization is unique.

Professor Barnard and Dr. Coral are to be congratulated upon the excellent presentation. Besides the general introduction, each chapter is preceded by an adequate summary and explanation of its contents. While Moore's notation is preserved throughout, the principal definitions and theorems are explained in words as well as in symbols, and everything possible has been done to make the book comprehensible to the reader. The difficult typography is excellently done.

Finally, it must not be forgotten that Barnard has himself made important contributions to the work which are more than editorial in character, although modesty has forbidden him to point these out.

\title{
C. C. MacDuffee
}

\section{REICHENBACH ON PROBABILITY}

Wahrscheinlichkeitslehre. By Hans Reichenbach. Leiden, Sijthoff, 1935. 451 pp.

A complete and perfect work on probability would tell what probability is, would give all mathematical developments in full, and would act as a guide to the application of the theory. The present work by Reichenbach has much of mathematical interest, and deals with a wide range of uses of the probability concept; but its greatest importance comes from the fact that it brings us definitely closer to an understanding of probability itself. This achievement is the more valuable in view of the many conflicting definitions which have been given. Reichenbach himself uses three definitions. The first is purely axiomatic; the second and third, frequency-limit and point-set definitions, are proved to be applications of the first. Other concepts, such as that based on equal probability or the psychological ones, he shows to be either untenable or reducible to the frequency-limit definition.

The author comes to the study of probability from the fields of both physics and philosophy. As a physicist he sees that we can not know that any rigorous laws rule the universe; nor yet-assuming this to be a world of law-the exact formulation of any law. Nor-assuming we knew a precise law to hold-can we measure with precision either the present state on which that law would base a prediction, or the future state which would verify it. So it is that a physicist, even though he ignore the added uncertainties introduced by quantum mechanics, finds the theory of probability essential to an understanding of our relations with nature. "We are dealing here, not alone with problems of 
mathematics and mathematical physics,-we are dealing with our ultimate knowledge of nature altogether, with the question of what our statements concerning reality actually mean." As a philosopher and logician, Reichenbach comes to his study of probability confident that a formalization of the theory is the best means for reaching clarity. Hence he begins with a brief but adequate account of formal logic and of the significance of a system of axioms-an account derived largely from Russell and Whitehead, Hilbert, and Ackermann.

The following chapter (Elementary calculus of probabilities) defines probability as a number, a function of two classes of events. The calculus is built up on axioms which, at many points, generalize the logical axioms of strict implication. Thus, in place of "the inclusion of event $x_{i}$ in class $O$ implies the inclusion of event $y_{i}$ in class $P$ with certainty," we have " $x_{i} \epsilon O$ implies $y_{i} \in P$ with probability $p$." In abbreviated symbolism $W(O, P)=p$. The definition and the symbol both emphasize the fact that probability depends not only on the conclusion but also on the premise; the probability that a die fall with the 6 uppermost depends on the set of throws used as a basis. Thus, consider the set of throws in which 6 is uppermost when the die is two inches above the table.

The axiomatic, abstract definition of probability does not assign to a particular pair of sequences a definite number-except that 1 and 0 are the values of the probability when $O$ implies $P$ and not- $P$ respectively. Neither does it afford us certainty that, for a given pair, the probability exists, nor even-in general-does it offer any criterion as to its existence. In fact, it is conceivable that various concrete definitions, each uniquely fixing the value of probability whenever it applied, would apply to different classes of sequence pairs.

The axioms were, naturally, devised in order to fit a particular concrete concept. They are satisfied if we define $W(O, P)$ as the limit of the ratio of the number of cases where $O$ and $P$ both hold to the total number of cases of $O$; and this is the only content given to the axioms so far as discrete sequences are concerned. Among the topics particularly well treated in the elementary discussion are Bayes' rule and the independence of events.

Many applications of the theory of probability depend, not only on the existence of probability for the given sequence, but also on the existence and value of that number for certain sub-sequences. Von Mises, in fact, goes so far as to deny the name of probability to sequences unless they satisfy a "principle of irregularity" which makes very sweeping demands of this nature. Reichenbach has analyzed this concept and found it untenable. He does, however, require of all infinite sequences yielding a probability that that number be unchanged by the omission of any finite number of terms at the beginning. He defines and uses frequently "normal sequences"- those in which a sub-sequence has the probability of the given sequence if inclusion of a term in the sub-sequence is determined either by the nature of other terms at fixed distances before it, or by its place in a regular rotation. Important abnormal sequences exist. Thus the positions of a gas molecule in an enclosed space at very short intervals will show after-effects. A brief study of such hereditary phenomena and of lattices, sequences of sequences, concludes the chapter.

In the short but adequate chapter on probability sequences in which the marks of the possible results of a trial are numbers-on mean values and 
variance - we may question the use of "Mittelwert" for the average of a finite number of quantities, "Durchschnitt" for that of an infinite sequence.

Reichenbach has given much attention to "geometric" probability-in which an additive measure of point sets may replace frequency and give a second concretion of the axioms. The chapter on this topic is valuable, both for its clear exposition of the concepts and for its variety of applications. This is a chapter, however, in which one could wish more care had been used. On page 225 there is mention of the "most probable value" in a continuum-since, in general, each value will have probability zero, the phrase, common as it is, should be avoided. Reichenbach, comparing surfaces of rotation with "Streckungsflächen" (p. 230) 一those of type $z=\phi_{1}(x) \phi_{2}(y)$-names the latter a more general type, implying to the unwary reader that they include the former. The reviewer would prefer that the book did not refer to "infinite summation of infinitesimal terms" (p. 239). On page 248 Reichenbach divides a time-interval $\tau$ into a finite number of sub-intervals; stating that the number of changes from $P$ to not- $P$ in $\tau$ equals the number of sub-intervals with $P$ at the beginning and not- $P$ at the end, he quite ignores the possibility of two or more reversals within a sub-interval.

Chapter 7, on Frequency properties of probability sequences, contains results notable for the combination of the axiomatic definition of probability and of the frequency concept. Thus Bernoulli's theorem assumes the following form: Given a sequence in which the probability of a certain event is $p$ and an arbitrary $\delta$, then the probability that the relative frequency of the event lie between $(p-\delta)$ and $(p+\delta)$ approaches 1 as $n$ becomes infinite. Thus, as an undefined probability occurs in the premise, another undefined probability replaces it in the conclusion-on a higher level, as it were. It should be noted that this is meaningless unless the sequence is immersed in a class of sequences.

For normal sequences Pólya has strengthened this theorem. According to him, 1 is the limit, not only of the probability that the $n$th relative frequency be in the interval named, but also of the probability that it remain there for all succeeding values of $n$. This is almost enough to identify the abstract probability with the limit of relative frequency. A further axiom is all that is needed in order to make this identification. The axiom is the plausible one: "If an event $C$ is to be expected in a sequence with a probability converging to 1 , it occurs in the sequence at least once." For normal sequences, then, which by definition have probabilities, the limit of the relative frequency must be the probability.

After a chapter on probabilities of higher type (probabilities of probabilities, dispersion and the like) come the concluding chapters, philosophical in nature, with valuable contributions to the question of the applicability of the theory to the world of experience. The chapters are entitled The problem of application and The logic of probability. "A scientific philosophy must require that every statement be verifiable." Have we a right, then, to say that the probability of an event is $2 / 3$ ? If we accept the frequency interpretation, we can not know, from the fin ite number of observations we can make, whether a limit is being approached; or, if so, whether that limit is $2 / 3$. We must be consoled - if it is consolation-by realizing that no measurement whatever can do more than this for us. Assuming, however, that we could know the prob- 
ability, how would that knowledge guide our action? For we act in a single case, on which probability has nothing to say. Reichenbach answers:-with a bet or an assumption. We act, he says, as if the outcome of highest probability were a certainty. This is, of course, too simple; if an aeroplane has $9 / 10$ probability of escaping disaster, many will nevertheless decline to be passengers. As for gaining an estimate of the value of the probability itself, Reichenbach sets up a rule - a decidedly weak rule - according to which we assume the probability to be near to the frequency already obtained. If a probability exists then this procedure will, in time, yield it within any required degree of accuracy-no one can say in how much time.

Moreover, the limit of frequency may not exist. If, for example, a "constant" in a natural law were to oscillate between two extremes in ever lengthening periods, the relative frequency of a phenomenon dependent on it might have no limit. Reichenbach mentions sequences similar to these-sequences, however, in which the transition between one probability and another is abrupt. These "comprehensible" (übersehbare) sequences are not sharply defined; yet our author holds that, if the sequences of nature are of this type, we can eventually prophesy future events with as much justification as if we had probability-sequences. Whether this is true can not be guessed until we have a careful definition; and once we had it, the thorough discussion of comprehensible sequences would require a treatise of length comparable to that of this book itself.

The conclusion as to the applicability of the theory of probability is this: We do not and cannot know whether we live in a world of sufficiently permanent lawfulness to justify prophesying the future on the basis of the past. Yet, past experience is the only guide to the future which we have; it may fail us, but reliance on it still remains our "best bet."

The book is not furnished with exercises for training the reader, but it is replete with illustrations from many fields of experience. Thus we have examples from physics, medicine, factory production, games of chance, astronomy, transportation, and so on. In some instances the notation should be clearer. Thus, on pages 260 and 272 the subscript $m$ in $H_{m}^{n}$ denotes the number of successes and $H$ itself is a proposition, whereas, on page $273, H_{n}\left(O, p^{k_{i}}\right)_{i}$ is a number, and the subscript the number of trials. In the last sentence of page $380, W\left(y_{1} \ni x_{1}\right)$ is the probability with which $y_{1}$ implies $x_{1}$, but $W\left(y_{1}\right)$ is the probability that $y_{1}$ is true.

By the omission of a few items (proofs concerning the Gaussian distribution, Stirling's formula, and Bernoulli's theorem, as well as tables of the probability integral) the author fails to make the book mathematically selfcontained. This is the more to be regretted, since completeness in these respects would have required so little additional space.

Reichenbach's book inevitably leaves many questions on the nature of probability still unanswered. But it marks such distinct advances in our knowledge concerning the concept that all mathematical and philosophical libraries should contain it; every thoughtful reader will be grateful for the new insight it brings to the subject. 